\title{
Apoptotic function of tumor-associated antigen RCAS1 in oral squamous cell carcinoma
}

\author{
Hideaki Tanaka', Takeshi Toyoshima ${ }^{1 *}, K^{1}$ enzo Sonoda², Ryoji Kitamura', Masaaki Sasaguri ${ }^{3}$, Shintaro Kawano ${ }^{1}$, \\ Ryota Matsubara', Yuichi Goto ${ }^{1}$ and Seiji Nakamura ${ }^{1}$
}

\begin{abstract}
Background: Receptor-binding cancer antigen expressed on SiSo cell (RCAS1) is derived from uterine adenocarcinoma and can induce apoptosis in lymphocytes, allowing tumor cells to escape from immune surveillance. RCAS1 is reportedly expressed in a membranous pattern on tumor cell or soluble one in serum of patients. The aim of this study was to investigate expression patterns of RCAS1 and the effect on apoptosis in oral squamous cell carcinoma (OSCC) cell lines.
\end{abstract}

Methods: In four kinds of OSCC cell lines (HSC-2, HSC-3, SQUU-A, and SQUU-B), RCAS1 mRNAs and proteins were determined by RT-PCR and immunocytochemistry. Membranous RCAS1 was determined by flow cytometry. Culture supernatants were analyzed for detection of soluble RCAS1 by dot blotting and enzyme-linked immunosorbent assay. Apoptotic ability of RCAS1 on the erythroid leukemia cell line K562 with the putative receptor was evaluated by flow cytometry in co-culture with highly metastatic SQUU-B, with knocked-down RCAS1 cells or in a no-cell contact condition.

Results: RCAS1 mRNA and proteins were expressed in all of OSCC cell lines. Membranous pattern were expressed in all cell lines, while soluble pattern was detected in all supernatants. RCAS1 mRNA, membranous and soluble RCAS1 were significantly seen in SQUU-B more than the other 3 cell lines $(P<0.05)$. K562 apoptosis was induced in co-culture with each of all cell lines, particularly with SQUU-B. Apoptosis was markedly reduced in co-culture with RCAS1 knockdown cells, but was induced in co-culture without cell contract of SQUU-B.

Conlusions: Our study suggests that RCAS1 has an apoptotic function via membranous/soluble expression pattern in OSCC cells. RCAS1 may thus affect tumor escape from immune surveillance in OSCC by inducing apoptosis.

Keywords: RCAS1, Oral squamous cell carcinoma, Apoptosis, Immune surveillance, Biomarker

\section{Background}

Receptor-binding cancer antigen expressed on SiSo cell (RCAS1) is recognized by the monoclonal antibody 22-1-1, and was initially identified in uterine adenocarcinoma $[1,2]$. Its expression is reportedly correlated with clinicopathological parameters in 15 human cancers, such as clinical stage, histological subtype, differentiation, tumor size, depth of invasion, lymphovascular space involvement, lymph node metastasis, and cytological results [3-20]. RCAS1 acts as a ligand for a putative receptor in cell lines of several human tissue types and peripheral lymphocytes, such as activated T, B, and NK cells. In vitro, RCAS1

\footnotetext{
* Correspondence: takesea@dent.kyushu-u.ac.jp

'Division of Maxillofacial Diagnostic and Surgical Sciences, Section of Oral and Maxillofacial Oncology, Faculty of Dental Science, Kyushu University, Maidashi 3-1-1, Higashi-ku, Fukuoka 812-8582, Japan

Full list of author information is available at the end of the article
}

inhibits growth of those activated cells and then induces their apoptosis [21]. Similarly, RCAS1 can induce apoptosis of tumor infiltrating lymphocytes (TILs) in cancer patients. This induction can lead to the escape of tumor cells from immune surveillance, thus contributing to tumor growth in vivo.

After proteolytic process, membranous RCAS1 is secreted to serum from tumor cells [22]. This process, also called "ectodomain shedding", affects activity of membrane protein by altering its localization and mode of action. For RCAS1, ectodomain shedding converts membranous protein to soluble one, which greatly influences its function. Soluble RCAS1 concentrations are reported via enzyme-linked immunosorbent assay (ELISA) to be significantly higher in patients with uterine cancer than in healthy blood donors [23]. Moreover, change of 
RCAS1 concentration during the culture was associated with response to treatments in patients with uterine and ovarian cancers. Compared with serum from healthy blood donors, serum from patients with uterine and ovarian cancers significantly inhibited growth of erythroid leukemia cell line K562 that express the putative RCAS1 receptor by inducing apoptosis [3]. Literature review revealed that RCAS1 could be considered as a biomarker since the expression was associated with important clinicopathological parameters for patient's management and prognosis [24]. Taken together, these data indicate that soluble RCAS1 could be a valuable biomarker for cancer, with the ability to improve insight into the disease state, to facilitate diagnosis, therapeutic strategies and disease monitoring, and to inhibit growth of receptor-expressing cells. Thus, detection of soluble RCAS1 in serum would be more useful than membranous RCAS1 as a clinical indicator, since the apoptotic ability of soluble RCAS1 can impact on the total ability of tumor cell more than that of a membranous one and blood sampling is clinically easy [22].

We previously reported that the expression of RCAS1 in oral squamous cell carcinoma (OSCC) was detected both in cytoplasm and on membrane of tumor cell in 41 of 130 cases $(31.5 \%)$ by immunohistochemistry. Moreover, the percentage of TUNEL positive TILs in patients with RCAS1 positive OSCC was significantly higher than in those with RCAS1 negative OSCC [25]. Furthermore, RCAS1 expression in oral epithelial dysplasia was related to earlier conversion to oral cancer [26]. However, no studies on RCAS1 expression patterns in OSCC have been reported. Moreover, the apoptotic function of RCAS1 in OSCC is also unclear. Therefore we examined expression kinetics of RCAS1 in OSCC cell lines, to whether or not expression depended on characteristics of tumor cell. We further investigated the apoptotic functions of membranous and soluble RCAS1 in OSCC cell lines.

\section{Methods}

\section{Cell lines}

Four human OSCC cell lines; HSC-2, HSC-3, SQUU-A, and SQUU-B, the uterine adenocarcinoma cell line SiSo as positive control and erythroid leukemia cell line K562 (which expresses the putative RCAS1 receptor) were used in the study. HSC-2 and HSC-3 were established from tumors of metastatic lymph nodes originating in oral squamous cell carcinomas of different patients [27].
SQUU-A and SQUU-B were established by our department from the same patient with recurrent tongue carcinoma using orthotopic implantation [28]. SQUU-B shows histologically invasive growth and has high metastatic potential $(86.7 \%$ incidence of cervical lymph node metastasis), whereas SQUU-A demonstrates expansive growth and has low metastatic ability. Cells were maintained in Dulbecco's modified Eagle's minimum essential medium (DMEM) supplemented with $10 \%$ fetal bovine serum (FBS) and incubated at $37^{\circ} \mathrm{C}$ in a $5 \% \mathrm{CO}_{2}$ atmosphere. Culture medium was changed on alternate days during the experiments. This study proposal was approved by the ethical committee of Kyushu University (No. 25-227).

\section{RNA extraction and reverse transcriptase-PCR}

OSCC and SiSo cells were cultured in $10 \mathrm{~cm}$ dishes to $70-80 \%$ confluence. Total RNA was then extracted from cultured cells that were harvested with a cell scraper using Sepasol-RNA Super G (Nacalai Tesque, Kyoto, Japan) according to the manufacturer's instructions. Primers were chosen through the GenBank sequence database (NCBI, Bethesda, MD, USA). Primer pairs for RCAS1 and glyceraldehyde-3-phosphate dehydrogenase $(G A P D H)$ are listed in Table 1. All oligonucleotides used as primers were synthesized by Genenet Co., Ltd (Fukuoka, Japan). After reverse transcription with isolated mRNA, reaction products were subjected to 28 cycles of PCR for $R C A S 1$ and $G A P D H$ at 15 seconds at $94^{\circ} \mathrm{C}$ for denaturation, 30 seconds at $60^{\circ} \mathrm{C}$ for annealing, and 60 seconds at $68^{\circ} \mathrm{C}$ for extension. PCR products were electrophoresed on $2 \%$ agarose gels. Bands were visualized by using ethidium bromide. Experiments were made in triplicate. Luminosity values were quantified using Image software (NIH, Bethesda, MD, USA). Mean values of triplicate measurements were calculated.

\section{Immunocytochemistry}

OSCC and SiSo cells were separately cultured on cover glasses to $50-60 \%$ confluence. Cultured cells were fixed in $75 \%$ methanol and then incubated with anti-mouse IgM RCAS1 antibody (MBL, Nagoya, Japan) for 2 hours. The cells were then incubated with secondary antibodies conjugated with anti-mouse IgM Alexa Fluor ${ }^{\circ} 488$ antibodies (Invitrogen, Carisbad, CA, USA) for 1 hour., and then counterstained with Hoechst 33342 (Molecular Probes, Eugene, Oregon, USA) for 5 minutes. Fluorescently

Table 1 Primer sequence and fragment size of RCAS1

\begin{tabular}{lll}
\hline & Forward primer & Reverse primer \\
\hline RCAS1 & 5'ATGGCCATCACCCAAGTTTCG-3' & 5'-TTATGAAAGTTCACACCAATT-3' \\
GAPDH & 5'TGACCTTGCCCACAGCCTT-3' & 5'-CATCACCATCTTCCAGGAGCG-3' \\
\hline
\end{tabular}


labeled cells were observed under a fluorescence microscope BZ-9000 (Keyence, Osaka, Japan).

\section{Flow cytometry}

OSCC and SiSo cells were separately cultured in $10-\mathrm{cm}$ dishes to $70-80 \%$ confluence. The cells were removed from dishes with $0.5 \%$ Trypsin/EDTA (Invitrogen) and collected by centrifugation (1200 rpm, $5 \mathrm{~min}$ ). Cultured cells were harvested and incubated on ice for $45 \mathrm{~min}$ with RCAS1 antibody. After washing, the cells were incubated on ice for $45 \mathrm{~min}$ with anti-mouse IgM Alexa Fluor 488 antibodies (Invitrogen), washed again and subjected to flow cytometry (FACSVerse, Becton Dickinson, San Jose, CA, USA). Isotype anti-mouse IgM antibodies (eBioscience, San Diego, CA, USA) was used as a negative control antibodies. Results were expressed as mean fluorescence intensity (MFI). Mean values of quadruplicate measurements are shown.

\section{Dot blotting}

OSCC and SiSo cells were separately cultured in $10-\mathrm{cm}$ dishes to $70-80 \%$ confluence. The culture supernatants were harvested for every 24-h incubation period from day 1 to day 4. Cell-free culture media treated under the same conditions was used as a control. To detect secreted RCAS1 in cell culture supernatants, chemiluminescent western dot blotting was performed. Briefly, each sample was applied to a nitrocellulose filter by a blotting apparatus (Rio-Rad, Richmond, CA, USA). Samples were treated with $3 \%$ hydrogen peroxide for $10 \mathrm{~min}$ at room temperature to remove endogenous peroxidase activity and air-dried. Next, filters were soaked in 5\% nonfat milk in Tris-buffered saline-Tween 20 (TBS-T) containing 5\% normal goat serum for $45 \mathrm{~min}$ at room temperature, then incubated with RCAS1 antibody in TBS-T containing 5\% normal goat serum for $1 \mathrm{~h}$ at room temperature. After washing three times with TBS-T, filters were incubated for $1 \mathrm{~h}$ with peroxidase conjugated goat anti-mouse IgM-HRP antibodies (Santa Cruz Biotechnology, Santa Cruz, CA, USA). After washing three times with TBS-T, filters were soaked with enhanced chemiluminescence reagents (SuperSignal West Pico Chemiluminescent Substrate ThermoScientific, Rockford, IL, USA) and visualized on X-ray films.

\section{ELISA}

OSCC and SiSo cells were cultured separately in $10-\mathrm{cm}$ dishes to $70-80 \%$ confluence. Media were changed, and the culture supernatants harvested, every after $24 \mathrm{~h}$ for days 1-4. Cell-free culture media treated under the same conditions was used as a control. In testing for soluble RCAS1 in OSCC cell lines, RCAS1 concentrations in culture supernatant were measured in triplicate with a RCAS1 ELISA kit (Cusabio Biotech Co., LTD, Wuhan,
Hubei Province, P.R. China), according to manufacturer's instructions. Mean concentrations of quadruplicate measurements are shown.

\section{siRNA transfection in SQUU-B}

Transfections were performed by using the X-tremeGENE siRNA transfection reagent (Roche, Mannheim, Germany), according to manufacturer's protocol. Briefly, SQUU-B cells were added to each well of a 6-well culture plate containing complete DMEM with 10\% FBS and incubated overnight for cell attachment. Unattached cells were then removed by washing and fresh DMEM medium (without antibiotics nor 10\% FBS) was added. Transfection reagent was mixed with RCAS1-siRNA (CST, Tokyo, Japan) or negative control siRNA (SigmaAldrich, lrvine, CA, USA) and incubated for 15 minutes at room temperature. The resulting complex was then added to each plate. After 2 days, medium was changed to new one containing 10\% FBS. The siRNA function was confirmed by RT-PCR, flow cytometry, and ELISA.

\section{Evaluation of apoptosis}

To measure the apoptotic effect of RCAS1 as a whole in K562, OSCC cells, SiSo and the RCAS1-knockdown SQUU-B cells were co-cultured with K562 cells in the 6well cell culture plate. To measure the effect of soluble RCAS1, SQUU-B cells and K562 cells were separately co-cultured in a 6-well culture plate with a cell culture insert (transparent PET membrane, $1.0-\mu \mathrm{m}$ pore size, BD Falcon, BD Biosciences, Franklin Lakes, NJ, USA). Then, $1 \times 10^{5}$ of each effector and K562 target cell were co-incubated in a 6 well-plate at effector/target $(\mathrm{E} / \mathrm{T}) \mathrm{ra}$ tios of 1:1, 5:1, 10:1, and 20:1. To discriminate K562 cells from effector cells, K562 cells were stained by the Green fluorescence cell linker PKH67 kit (Sigma, St.Louis, MO, USA) before initiating the co-culture. Suspended cells were harvested and stained with Annexin V-PE apoptosis detection kit (eBioscience) on days 1-4 after the experiment. Flow cytometry was used to count apoptotic cells after Annexin V-PE staining on days 1-4.

\section{Statistical analysis}

We assessed differences in RCAS1 expression and secretion, and the Annexin-V positive ratio between different cell groups with the Mann-Whitney $U$-test. $P<0.05$ was considered to be statistically significant.

\section{Results}

Expression of RCAS1 mRNA and protein in OSCC cell lines RT-PCR results revealed that RCAS1 mRNA was detected in all OSCC cell lines as in the positive control, SiSo (Figure 1A). Luminosity analysis revealed that highly metastatic SQUU-B significantly expressed more RCAS1 mRNA than the other 3 OSCC cells (Figure 1B). 

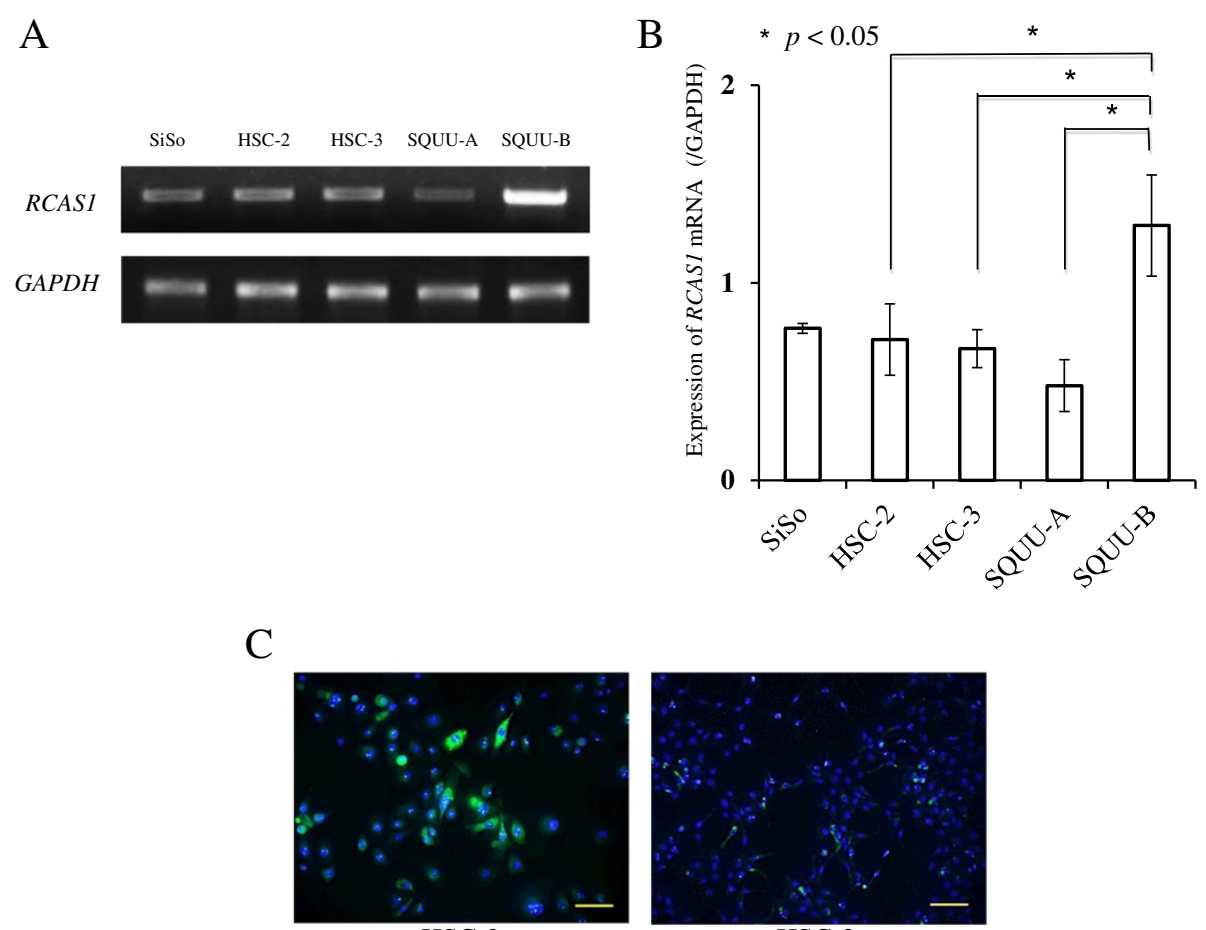

HSC-2

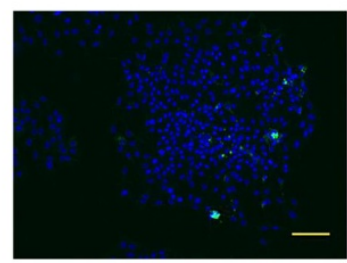

SQUU-A
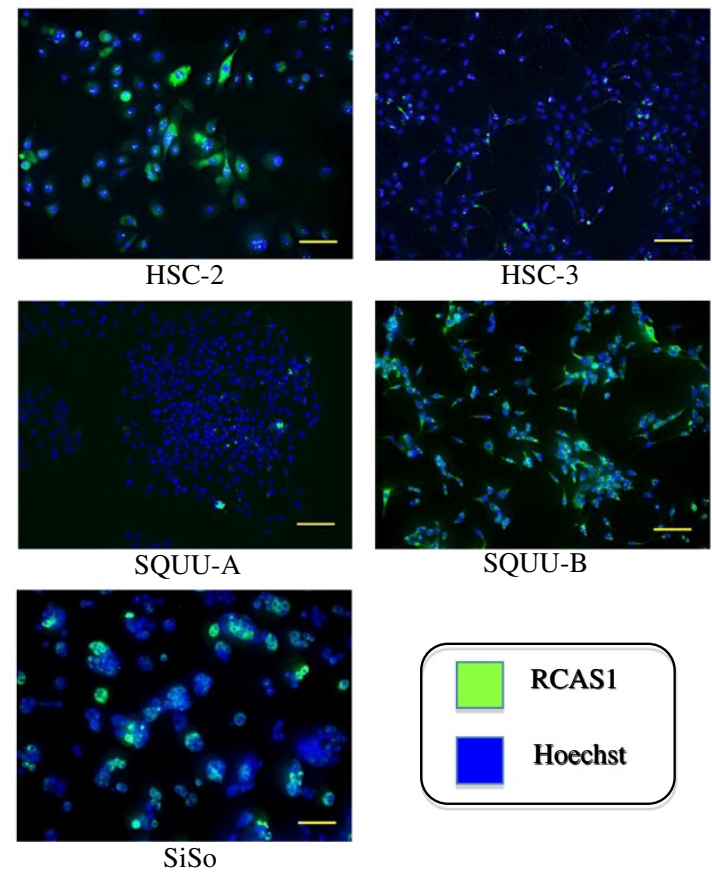

HSC-3

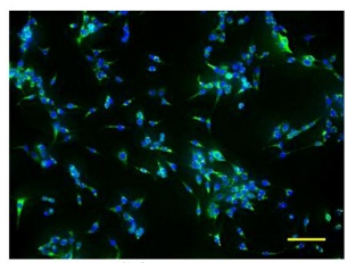

SQUU-B

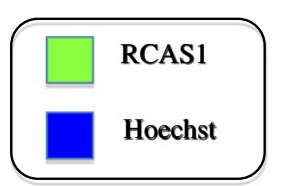

Figure 1 RCAS1 expression in OSCC cell lines (HSC-2, HSC-3, SQUU-A, SQUU-B) and SiSo (uterine adenocarcinoma cells; positive control) using RT-PCR and immunocytochemistry. A RCAS1 mRNA was firmly detected in all OSCC cell lines. B Analysis for luminosity (Image J software) revealed that RCAS1 mRNA of highly metastatic SQUU-B significantly scored higher than did the other 3 OSCC cells $(P<0.05$; Mann-Whitney U-test). Mean values of triplicate measurements are shown. C RCAS1 protein was diffusely expressed in both cytoplasm and on membranes in all OSCC cell lines and SiSo, and was especially strong in SQUU-B cells (magnification × 10; bar: $100 \mu \mathrm{m}$ ).

Immunocytochemical results revealed that RCAS1 proteins were diffusely expressed both in cytoplasm and membrane of all OSCC cell lines as in SiSo, and was more strongly expressed in SQUU-B (Figure 1C).

\section{Expression of membranous RCAS1 in OSCC cell lines}

Since RCAS1 is reportedly found in membranous and soluble patterns in several cancers, we first evaluated patterns of RCAS1 expression in OSCC cell lines. Flow cytometric analyses revealed membranous RCAS1 to be expressed in all OSCC cell lines (Figure 2A). Results were statistically compared by mean fluorescence intensity (MFI). MFI of membranous RCAS1 was significantly higher in SQUU-B $(1733.8 \pm 764.9)$ than in the other OSCC cells: HSC-2 (317.6 \pm 68.2$)$, HSC-3 (133.3 \pm 36.62$)$, and SQUU-A $(135.0 \pm 36.1)(P<0.05$; Mann-Whitney $U$ test; Figure $2 \mathrm{~B})$.

\section{Detection of soluble RCAS1 in supernatant of OSCC cells}

Culture supernatants of OSCC cells were next examined for soluble RCAS1. Results of dot blotting revealed that soluble RCAS1 in culture supernatants was detected in 


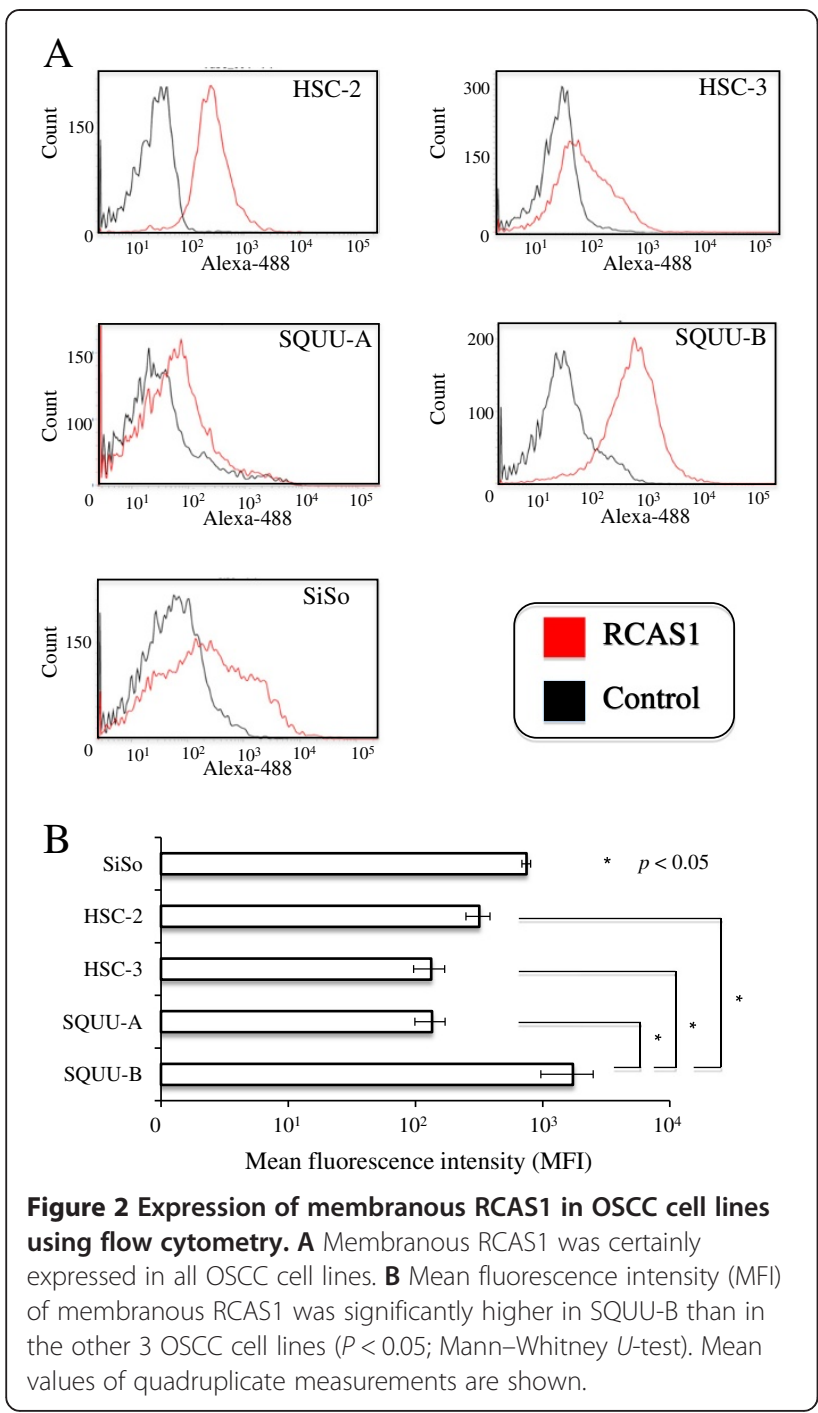

all OSCC cell lines, which increased in amount over the culture period (Figure 3A). ELISA analyses found values of soluble RCAS1 to be $49.7 \pm 1.0 \mathrm{U} / \mathrm{ml}$ for HSC-2, $55.31 \pm 1.2 \mathrm{U} / \mathrm{ml}$ for HSC-3, $18.1 \pm 2.4 \mathrm{U} / \mathrm{ml}$ for SQUUA, and $64.7 \pm 0.5 \mathrm{U} / \mathrm{ml}$ for SQUU-B by day 4 , while were $34.6 \pm 1.21 \mathrm{U} / \mathrm{ml}$ for $\mathrm{HSC}-2,33.8 \pm 1.2 \mathrm{U} / \mathrm{ml}$ for HSC-3, $6.7 \pm 0.4 \mathrm{U} / \mathrm{ml}$ for SQUU-A, and $41.9 \pm 0.3 \mathrm{U} / \mathrm{ml}$ for SQUU-B by day 1 . The SQUU-B supernatant significantly carried more soluble RCAS1 than the other OSCC cell lines by day $4(P<0.05$; Mann-Whitney $U$ test, Figure 3B).

\section{Apoptosis of K562 cells induced by RCAS1 from OSCC cells}

RCAS1-induced apoptosis in K562 cells was analyzed using a co-culture system with OSCC cells. Each OSCC cell line induced apoptosis in $\mathrm{K} 562$ cells. The Annexin V positive ratio of $\mathrm{K} 562$ increased with $\mathrm{E} / \mathrm{T}$ ratios and culture periods under all co-culture conditions with each

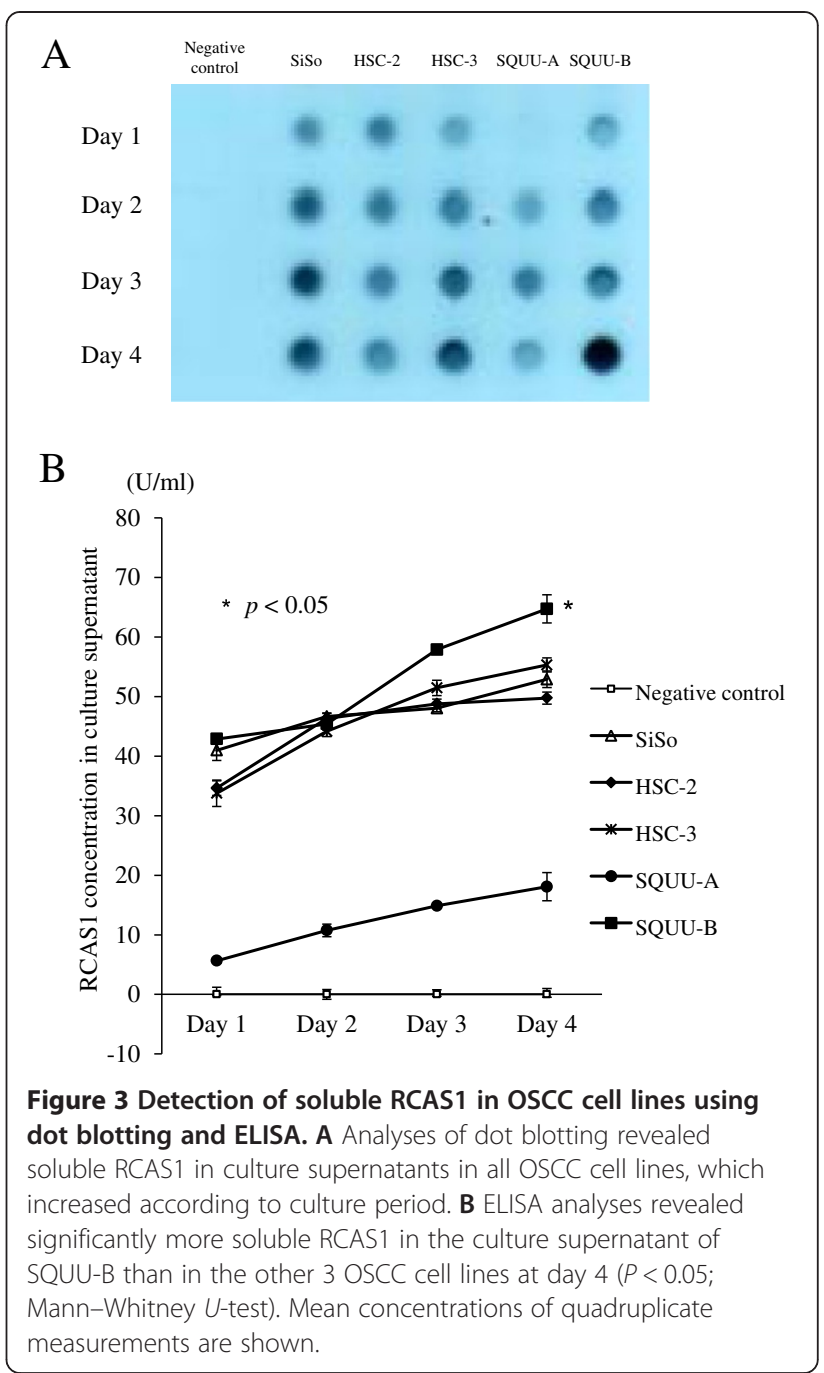

OSCC cell line. As representative results, the ratio increased in an $\mathrm{E} / \mathrm{T}$ ratio-dependent fashion by co-culture day $4 ; 28.4 \%$ with HSC-2, $16.6 \%$ with HSC-3, $10.8 \%$ with SQUU-A, and 52.5\% with SQUU-B at a 20:1 E/T ratio. Apoptosis of $\mathrm{K} 562$ cells were more strongly induced by co-culture with SQUU-B cells than the other 3 OSCC cell lines (Figure 4).

Next, the same assay was conducted under different conditions of co-culture with RCAS1 knockdown SQUU-B cells and no-contact co-culture using a cell culture insert. First, results of RT-PCR, flow cytometry, and ELISA confirmed that the RCAS1 siRNA transfection was successful and the RCAS1 was silenced (Figure 5A-C). When K562 cells were cultured with SQUU-B as positive control, the Annexin- $\mathrm{V}$ positive ratios for $\mathrm{K} 562$ were $1.5 \%$ at a 5:1 E/T ratio, $2.2 \%$ at $10: 1$ and $3.0 \%$ at $20: 1$ by day $1 ; 5.22 \%$ at $5: 1$, $15.0 \%$ at $10: 1$, and $18.4 \%$ at $20: 1$ by day $2 ; 19.2 \%$ at $5: 1$, $25.9 \%$ at $10: 1$ and $41.7 \%$ at $20: 1$ by day 3 ; and $21.3 \%$ at $5: 1$, $29.7 \%$ at $10: 1$ and $50.5 \%$ at 20:1 by day 4 . When K562 cells were cultured with RCAS1 knockdown SQUU-B cells, 


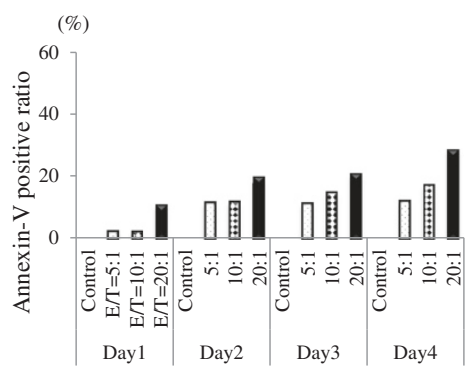

HSC-2
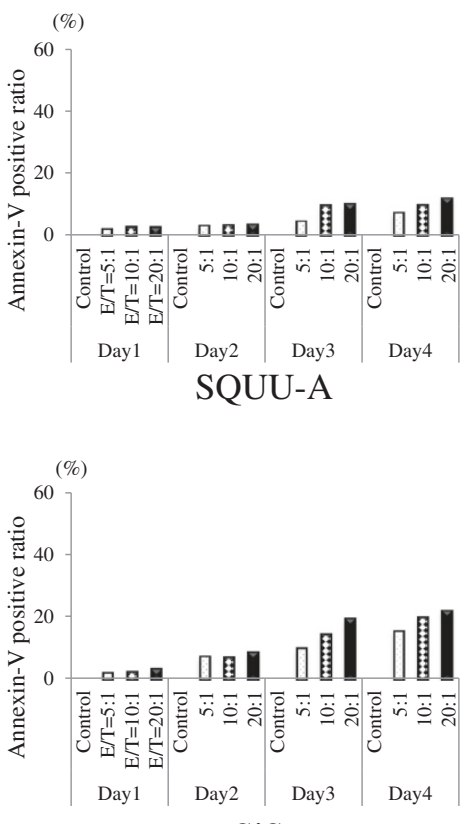

SiSo

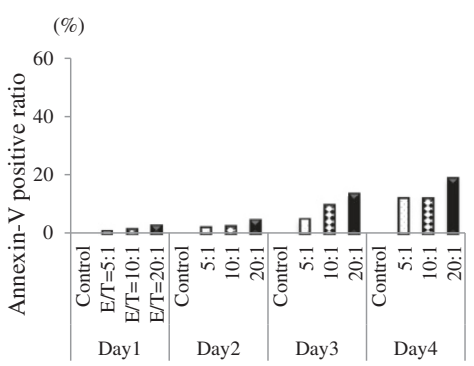

HSC-3

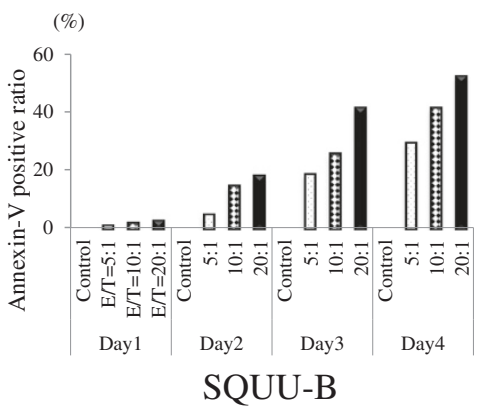

Figure 4 Representative results for Annxin-V positive ratio of K562 cultured with OSCC cell lines. Apoptosis rates for K562 cells gradually increased by $\mathrm{E} / \mathrm{T}$ ratios and culture periods in all conditions of co-culture for each OSCC cell lines. Apoptosis of K562 was more induced in the co-culture with SQUU-B cells than with the other 3 OSCC cell lines.

Annexin-V positive ratio of $\mathrm{K} 562$ were $0.2 \%$ at $5: 1,0.5 \%$ at $10: 1$, and $0.6 \%$ at $20: 1$ by day $1 ; 0.3 \%$ at $5: 1,0.7 \%$ at $10: 1$, and $1.3 \%$ at $20: 1$ by day $2 ; 3.2 \%$ at $5: 1,5.6 \%$ at $10: 1$, and $5.1 \%$ at $20: 1$ by day 3 ; and $3.4 \%$ at $5: 1,6.7 \%$ at $10: 1$ and $10.8 \%$ at $20: 1$ by day 4 . When co-cultured with the RCAS1-knockdown cells, $41.7 \%$ of the Annexin-V positive cells were inhibited at a 20:1 E/T ratio by day 4. Apoptosis of $\mathrm{K} 562$ cells was remarkably inhibited when co-cultured with RCAS1 knockdown SQUU-B cells through the culture periods (Figure 6).

Even when K562 cells were cultured with SQUU-B without cell contact, using the cell culture insert, the K562 Annexin-V positive ratio measured by flow cytometry showed apoptosis increased due to culture periods and $\mathrm{E} / \mathrm{T}$ ratios: $0.7 \%$ at $5: 1,1.0 \%$ at $10: 1$, and $3.8 \%$ at $20: 1$ by day $1 ; 3.9 \%$ at $5: 1,7.0 \%$ at $10: 1$, and $11.4 \%$ at $20: 1$ by day $2 ; 6.3 \%$ at $5: 1,9.4 \%$ at $10: 1$, and $18.5 \%$ at $20: 1$ by day $3 ; 11.4 \%$ at $5: 1,21.3 \%$ at $10: 1,31.5 \%$ at $20: 1$ by day 4. Thus, apoptosis of K562 cells was induced when cultured with only soluble RCAS1 (Figure 6).

\section{Discussion}

To our knowledge, this is the first report to find membranous and soluble RCAS1 in OSCC. Expression of RCAS1 mRNA in OSCC cells accords with that in 10 kinds of breast cancer cells [29] and 4 kinds of cholangiocarcinoma cells [30]. Moreover, expression of RCAS1 protein in OSCC cells accords with that in 4 kinds of breast cancer cells [31]. These consistencies encouraged us to further investigate its expression patterns, as immunocytochemical analysis individual cannot distinguish between membranous and soluble RCAS1.

Results of flow cytometry for membranous RCAS1 in OSCC cells are congruent with those for SiSo and TMCC (uterine cervical cancer), $\mathrm{MH}$ and Kuramochi (ovarian mucinous cancer), KF, HRA (ovarian serous 


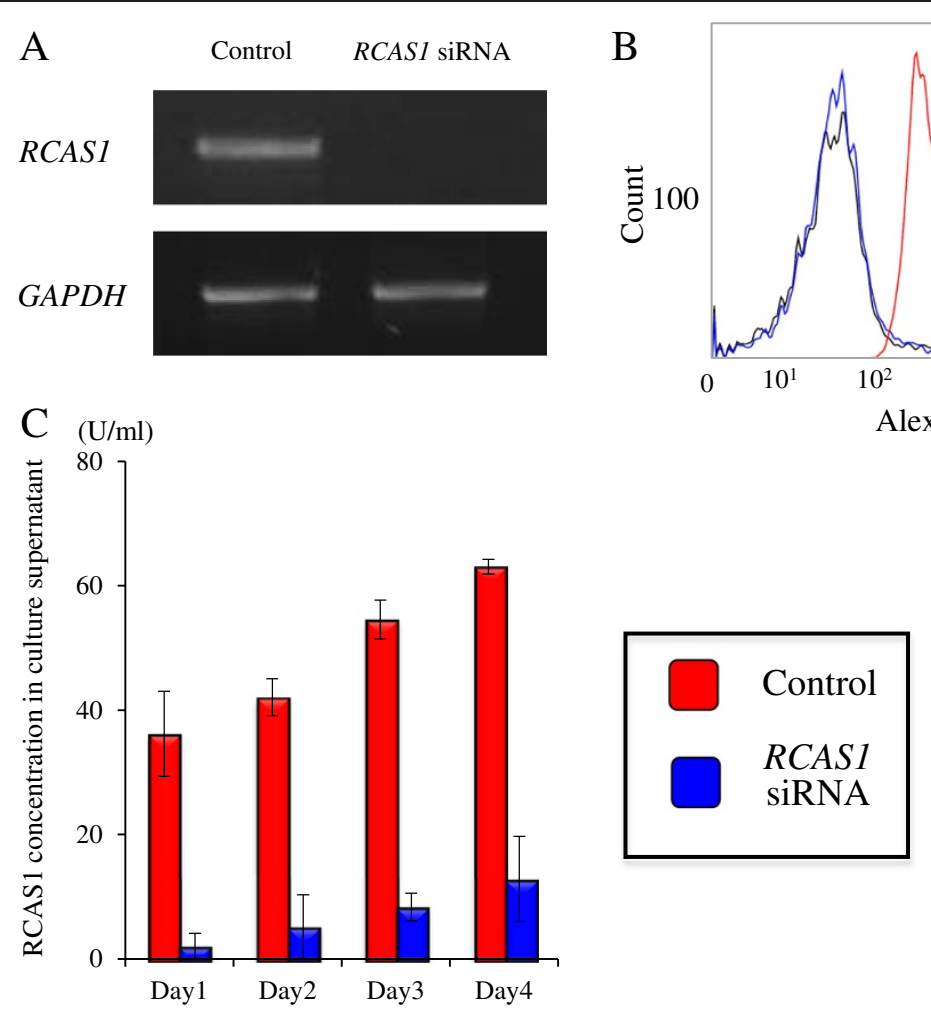

Figure 5 RCAS1 siRNA transfection in SQUU-B. A RT-PCR analyses revealed that RCAS1 siRNA successfully reduced RCAS1 mRNA levels in SQUU-B. B Flow cytometric analyses revealed that RCAS1 siRNA certainly down-regulated the expression of membranous RCAS1 in SQUU-B. C ELISA analyses revealed that RCAS1 siRNA definitely down-regulated the soluble RCAS1 in SQUU-B through the culture periods. Mean concentrations of quadruplicate measurements are shown.

cancer), and MCF-7 (breast cancer) [2,32]. Results of the dot blotting and ELISA suggest that the soluble RCAS1 could be definitely detected in all of OSCC cells and its concentrations were time-dependent. These results align with findings that soluble RCAS1 in serum is significantly increased in colon cancer patients compared with that in healthy individuals; higher concentration of soluble RCAS1 is significantly associated with advanced Dukes' stage and high histopathological tumor grade; colon cancer patients with the higher concentration had significantly shorter overall survival time; and soluble RCAS1 is an independent prognostic factor for this malignancy $[33,34]$.

Interestingly, the significant luminosity of RCAS1 mRNA and the significant MFI value of membranous RCAS1 in SQUU-B suggest that RCAS1 expression is associated with its high metastatic potential, as does its significant increase in soluble RCAS1 levels by day 4 . Therefore, highly metastatic OSCC cells are likely to express RCAS1 as membranous or soluble proteins. In cervical and ovarian cancers, the number of cells that express mesenchymal marker vimentin is inversely related to RCAS1 expression, as remodeling of stromal tissue by vimentin results in tumor invasion and metastasis
[35]. Moreover, enhanced RCAS1 expression in African Green Monkey kidney fibroblast cell lines COS-7 following introduction of the RCAS1 gene significantly promotes in vivo tumor growth via increase of vascular endothelial growth factor [36], which implies that RCAS1 affects tumor progression through lymph nodal metastasis. Combined with the significant expression of RCAS1 in SQUU-B with high metastatic potential, those findings suggest that RCAS1 may play a pivotal role in tumor progression by lymph nodal metastasis.

Since both results of apoptotic cell detection by staining of Annexin $\mathrm{V}$ and propidium iodide were synchronized in vitro [21] and we could confirm that apoptosis of tumor-infiltrating lymphocytes was found by TUNNEL staining in vivo [25], the staining of Annexin $\mathrm{V}$ individual was used for the detection in this study. Results of flow cytometry indicate that apoptosis of $\mathrm{K} 562$ cells is markedly induced in the co-culture system with each OSCC cells, and this incidence was dependent on RCAS1 concentration and culture periods. These results do not align with reports that K562 apoptosis was not induced in co-culture with MCF-7 breast cancer cells [22]. Therefore, to assess the extent of this apoptotic ability from soluble RCAS1, we used RCAS1 siRNA transfection to create an RCAS1- 


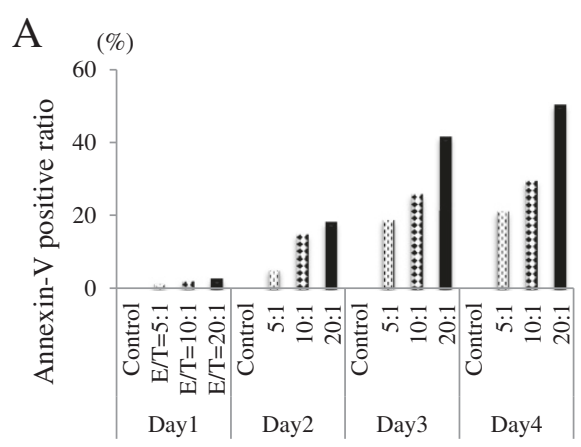

Control

C $(\%)$

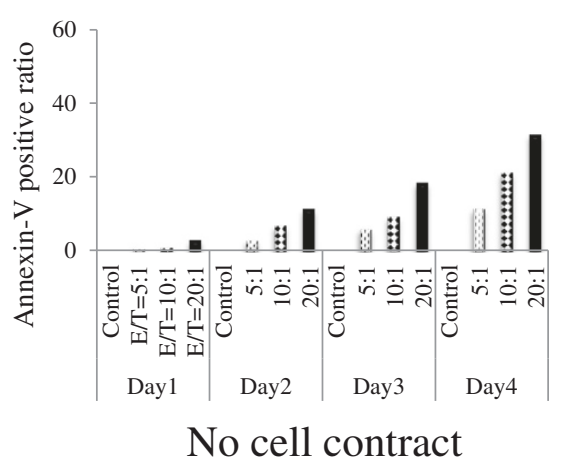

B

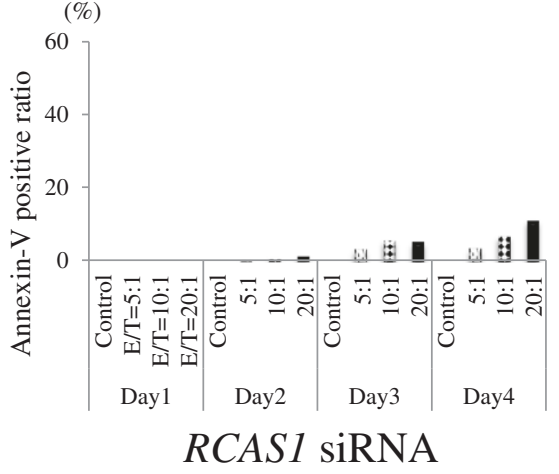

Figure 6 Representative results for Annxin-V positive ratio of K562 via membranous and soluble RCAS1 in SQUU-B. A Annexin- $V$ positive ratio of K562 cultured with SQUU-B (positive control). Apoptotic rates for K562 cells gradually increased according to E/T ratios and culture periods. B With the RCAS1 siRNA transfection, apoptosis of K562 was remarkably inhibited. C In the co-culturing of K562 cells with SQUU-B without cell contact (using a cell culture insert), K562 apoptosis was still induced.

silenced SQUU-B with high metastatic potential. We then co-cultured K562 cells in medium shared with either RCAS1-silenced or SQUU-B without direct cell contact. By day 4 at a $20: 1 \mathrm{E} / \mathrm{T}$ ratio, the Annexin- $\mathrm{V}$ positive ratio was $31.5 \%$ for K562 cells cultured with SQUU-B without direct contact, and $50.5 \%$ in $\mathrm{K} 562$ cells cultured in contact with SQUU-B, which suggests that $31.5 \%$ was induced by soluble RCAS1 versus $19.0 \%$ by membranous RCAS1. Since membranous/soluble RCAS1 value from SQUU-B was absolutely higher than that from positive control SiSo and SQUU-B is specific with high metastatic potential, it might be possible that even membranous RCAS1 had the apoptotic ability in this study. Moreover, soluble RCAS1 apparently affects apoptotic ability of OSCC cell more than membranous RCAS1. Though apoptotic ability of membranous and soluble RCAS1 in K562 was merely analyzed in this study, it has been reported that the cell growth of $\mathrm{K} 562$ was inhibited, after the culture for 72 hours with the serum including soluble RCAS1 of more than $10 \mathrm{U} / \mathrm{ml}$ from patients with uterine cancer [23]. In addition, it has been also reported that the apoptosis of activated T cells by IL-2 was markedly induced after culture for 72 hours with recombinant RCAS1- glutathione S-transferase fusion proteins, while the apoptosis of CD3-positive (non-activated) T cells was not [21]. Combined these results with our findings, though immune system of patient initially finds cancer occurred via $T$ cell recognition and TILs attack the cancer to prevent from the growth and the invasion, RCAS1 could finally induce apoptosis of TILs such as T cells in patients with OSCC. We previously reported that patients with RCAS1 positive OSCCs have significantly higher percentages of TUNEL positive TILs than do patients with RCAS1 negative OSCCs [25]. These results accord with reports that TIL apoptosis rates in colorectal [10] and gastric carcinomas [13] were significantly higher in RCAS1 positive tissues than in RCAS1 negative tissues. Therefore, RCAS1induced apoptosis of TILs in patients with OSCC can lead to escape of tumor cells from immune surveillance and contribute to tumor growth in vivo.

RCAS1 could be defined as tumor-associated antigen in OSCC as well as in other cancers. There could be clarified to be two patterns of RCAS1 expression in OSCC and the apoptotic ability of soluble RCAS1 could impact on the total ability of tumor cell more than that of membranous one like other cancers. In addition, the 
possibility of RCAS1 has been proposed as a biomarker of ovarian and uterine cancer $[3,23]$. Thus, detection of soluble RCAS1 in serum of patients with OSCC might be available as a biomarker of OSCC to facilitate diagnosis, therapeutic strategies and disease monitoring, as well as improving insight into the disease state. If so, RCAS1 could be applicable for OSCC treatment as a targeted molecule. Several strategies for RCAS1 could be considered such as modulation of RCAS1 with siRNA or inhibition of RCAS1 with monoclonal antibody, in order to suppress its expression and function. The identification of useful biomarkers is crucial and recent advances in biomarker discovery have raised new opportunities in the emerging fields of personalized and predictive medicine. Further clinical studies are warranted to investigate soluble RCAS1 in serum of patients with OSCC and to clarify the association of RCAS1 expression with clinicopathological parameters.

\section{Conclusion}

RCAS1 has an apoptotic function via membranous and soluble expression in OSCC cells. Moreover, soluble RCAS1 could extremely impact on the ability, compared to membranous one. RCAS1 may thus affect tumor escape from immune surveillance in OSCC by inducing apoptosis.

\begin{abstract}
Abbreviations
DMEM: Dulbecco's modified Eagle's minimum essential medium; E/T: Effector/ target ratio; ELISA: Enzyme-linked immunosorbent assay; FBS: Fetal bovine serum; GAPDH: Glyceraldehyde-3-phosphate dehydrogenase; MFI: Mean fluorescence intensity; OSCC: Oral squamous cell carcinoma; RCAS1: Receptorbinding cancer antigen expressed on SiSo cell; TBS-T: Tris-buffered saline-Tween 2; TILs: Tumor infiltrating lymphocytes.
\end{abstract}

\section{Competing interests}

All authors have no financial or personal relationships with other people or organisations that could inappropriately influence (bias) their work.

\begin{abstract}
Authors' contributions
HT carried out mainly the all experiments and described the manuscript. TT especially designed RT PCR, immunocytochemistry, and siRNA transfection and described mainly the manuscript. KS especially designed flow cytometry, dot blotting, and ELISA, and provided materials. RK participated in statistical analyses. MS provided materials and participated in the study design. SK participated in the study design and helped to draft this manuscript. RM participated in the establishment of OSCC cell lines and siRNA transfection. YG participated in the establishment of OSCC cell lines, RT PCR, and immunocytochemistry. SN helped to draft this manuscript. All authors have revised this manuscript and agreed for submission.
\end{abstract}

\section{Acknowledgment}

The work was supported by a Grant-in-Aid for scientific research from the Japanese Ministry of Education, Culture, Science, Sports and Technology of Japan (No. 24249091, 25861955).

\section{Author details}

'Division of Maxillofacial Diagnostic and Surgical Sciences, Section of Oral and Maxillofacial Oncology, Faculty of Dental Science, Kyushu University, Maidashi 3-1-1, Higashi-ku, Fukuoka 812-8582, Japan. ${ }^{2}$ Department of Obstetrics and Gynecology, Graduate School of Medical Sciences, Kyushu University, Fukuoka, Japan. ${ }^{3}$ Department of Science of Physical Functions, Division of Oral and Maxillofacial Surgery, Kyushu Dental University, Kitakyushu, Japan.
Received: 9 February 2014 Accepted: 28 April 2014

Published: 6 May 2014

\section{References}

1. Sonoda K, Nakashima M, Saito T, Amada S, Kamura T, Nakano H, Watanabe $\mathrm{T}$ : Establishment of a new human uterine cervical adenocarcinoma cellline, siso, and its reactivity to anticancer reagents. Int J Oncol 1995, 6:1099-1104.

2. Sonoda K, Nakashima M, Kaku T, Kamura T, Nakano H, Watanabe T: A novel tumor-associated antigen expressed in human uterine and ovarian carcinomas. Cancer 1996, 77:1501-1509.

3. Sonoda K, Miyamoto S, Yotsumoto F, Yagi H, Nakashima M, Watanabe T, Nakano $\mathrm{H}$ : Clinical significance of RCAS1 as a biomarker of ovarian cancer. Oncol Rep 2007, 17:623-628.

4. Iwasaki T, Nakashima M, Watanabe T, Yamamoto S, Inoue $Y$, Yamanaka $H$, Matsumura A, luchi K, Mori T, Okada M: Expression and prognostic significance in lung cancer of human tumor-associated antigen RCAS1. Int J Cancer 2000, 89:488-493.

5. Sonoda K, Kaku T, Hirakawa T, Kobayashi H, Amada S, Sakai K, Nakashima M, Watanabe T, Nakano $\mathrm{H}$ : The clinical significance of tumor-associated antigen RCAS1 expression in the normal, hyperplastic, and malignant uterine endometrium. Gynecol Oncol 2000, 79:424-429.

6. Umeoka K, Sanno N, Oyama K, Tahara S, Kurotani R, Ikuyama S, Nakashima M, Watanabe T, Osamura RY, Teramoto A: Immunohistochemical analysis of RCAS1 in human pituitary adenomas. Mod Pathol 2001, 14:1232-1236.

7. Hiraoka K, Hida Y, Miyamoto M, Oshikiri T, Suzuoki M, Nakakubo Y, Shinohara T, Itoh T, Shichinohe T, Kondo S, Kasahara N, Katoh H: High expression of tumor-associated antigen RCAS1 in pancreatic ductal adenocarcinoma is an unfavorable prognostic marker. Int J Cancer 2002, 99:418-423.

8. Oizumi S, Yamazaki K, Nakashima M, Watanabe T, Hommura F, Ogura S, Nishimura M, Dosaka-Akita H: RCAS1 expression: a potential prognostic marker for adenocarcinomas of the lung. Oncology 2002, 62:333-339.

9. Akashi T, Oimomi H, Nishiyama K, Nakashima M, Arita Y, Sumii T, Kimura T, Ito T, Nawata H, Watanabe T: Expression and diagnostic evaluation of the human tumor-associated antigen RCAS1 in pancreatic cancer. Pancreas 2003, 26:49-55.

10. Okada K, Nakashima M, Komuta K, Hashimoto S, Okudaira S, Baba N, Hishikawa Y, Koji T, Kanematsu T, Watanabe T: Expression of tumorassociated membrane antigen, RCAS1, in human colorectal carcinomas and possible role in apoptosis of tumor-infiltrating lymphocytes. Mod Pathol 2003, 16:679-685.

11. Sonoda K, Miyamoto S, Hirakawa T, Kaku T, Nakashima M, Watanabe T, Akazawa K, Fujita T, Nakano H: Association between RCAS1 expression and clinical outcome in uterine endometrial cancer. Br J Cancer 2003, 89:546-551.

12. Muta K, Ohshima K, Abe Y, Uike N, Choi I, Matsushima T, Nishimura J, Kikuchi M, Nakashima M, Watanabe T, Nawata H: Expression of human tumor-associated antigen RCAS1 in adult T-cell leukemia/lymphoma. Int J Hematol 2004, 79:340-344.

13. Nakamura Y, Yamazaki K, Oizumi S, Nakashima M, Watanabe T, Dosaka-Akita $H$, Nishimura M: Expression of RCAS1 in human gastric carcinoma: a potential mechanism of immune escape. Cancer Sci 2004, 95:260-265.

14. Leelawat K, Engprasert S, Tujinda S, Suthippintawong C, Enjoji M, Nakashima M, Watanabe T, Leardkamolkarn V: Receptor-binding cancer antigen expressed on SiSo cells can be detected in metastatic lymph nodes from gastrointestinal cancers. World J Gastroenterol 2005, 11:6014-6017.

15. Coban S, Ozkan H, Koklu S, Yuksel O, Kockar MC, Akar T, Ormeci N: The utility of serum receptor-binding cancer antigen expressed on SiSo cells in gastrointestinal tract cancers. Can J Gastroenterol 2006, 20:593-596.

16. Kohri T, Sugano M, Kawashima O, Saito R, Sakurai S, Sano T, Nakajima T: Prognostic model of stage II non-small cell lung cancer by a discriminant analysis of the immunohistochemical protein expression. Surg Today 2006, 36:1039-1046.

17. Tsujitani S, Saito H, Oka S, Sakamoto T, Kanaji S, Tatebe S, Ikeguchi M: Prognostic significance of RCAS1 expression in relation to the infiltration of dendritic cells and lymphocytes in patients with esophageal carcinoma. Dig Dis Sci 2007, 52:549-554.

18. Giaginis C, Davides D, Zarros A, Noussia O, Zizi-Serbetzoglou A, Kouraklis G, Theocharis S: Clinical significance of tumor-associated antigen RCAS1 
expression in human pancreatic ductal adenocarcinoma. Dig Dis Sci 2008, 53:1728-1734

19. Yang YC, Zhao YP, Liao Q, Zhang TP, Hu Y, Dai MH: Evaluation of RCAS1 as serum tumor marker for pancreatic cancer. Zhonghua Wai Ke Za Zhi 2009, 47:999-1001.

20. Theocharis S, Klijanienko J, Giaginis C, Rodriguez J, Jouffroy T, Girod A, Tsourouflis G, Sastre-Garau X: RCAS1 expression in mobile tongue squamous cell carcinoma: an immunohistochemical study. Med Sci Monit 2011, 17:Br228-Br234.

21. Nakashima M, Sonoda K, Watanabe T: Inhibition of cell growth and induction of apoptotic cell death by the human tumor-associated antigen RCAS1. Nat Med 1999, 5:938-942.

22. Sonoda K, Miyamoto S, Nakashima M, Wake N: Receptor-binding cancer antigen expressed on SiSo cells induces apoptosis via ectodomain shedding. Exp Cell Res 2010, 316:1795-1803.

23. Sonoda K, Miyamoto S, Hirakawa T, Yagi H, Yotsumoto F, Nakashima M, Watanabe T, Nakano H: Clinical significance of RCAS1 as a biomarker of uterine cancer. Gynecol Oncol 2006, 103:924-931.

24. Giaginis C, Giagini A, Theocharis S: Recepter-binding cancer antigen expressed on SiSo cells (RCAS1): a novel biomarker in the diagnosis and pregnosis of human neoplasia. Histol Histopathol 2009, 24:761-776

25. Toyoshima T, Nakamura S, Kumamaru W, Kawamura E, Ishibashi H, Hayashida JN, Moriyama M, Ohyama Y, Sasaki M, Shirasuna K: Expression of tumor-associated antigen RCAS1 and its possible involvement in immune evasion in oral squamous cell carcinoma. J Oral Pathol Med 2006, 35:361-368.

26. Tsai TC, Yu CH, Cheng SJ, Liu BY, Chen HM, Chiang CP: Expression of RCAS1 is significantly associated with the progression and prognosis of oral squamous cell carcinomas in Taiwan. Oral Oncol 2008, 44:759-766.

27. Momose F, Araida T, Negishi A, Ichijo H, Shioda S, Sasaki S: Variant sublines with different metastatic potentials selected in nude mice from human oral squamous cell carcinomas. J Oral Pathol Med 1989, 18:391-395.

28. Morifuji M, Taniguchi S, Sakai H, Nakabeppu Y, Ohishi M: Differential expression of cytokeratin after orthotopic implantation of newly established human tongue cancer cell lines of defined metastatic ability. Am J Pathol 2000, 156:1317-1326.

29. Tsuneizumi M, Emi M, Nagai H, Harada H, Sakamoto G, Kasumi F, Inoue S, Kazu T, Nakamura Y: Overrepresentation of the EBAG9 gene at 8q23 associated with early-stage breast cancers. Clin Cancer Res 2001, 7:3526-3532.

30. Enjoji M, Nakashima M, Nishi H, Choi I, Oimomi H, Sugimoto R, Kotoh K, Taguchi K, Nakamuta M, Nawata H, Watanabe T: The tumor-associated antigen, RCAS1, can be expressed in immune-mediated diseases as well as in carcinomas of biliary tract. J Hepatol 2002, 36:786-792.

31. Rousseau J, Tetu B, Caron D, Malenfant P, Cattaruzzi P, Audette M, Doillon C, Tremblay JP, Guerette B: RCAS1 is associated with ductal breast cancer progression. Biochem Biophys Res Commun 2002, 293:1544-1549.

32. Reimer TA, Anagnostopoulos I, Erdmann B, Lehmann I, Stein H, Daniel P, Dorken B, Rehm A: Reevaluation of the 22-1-1 antibody and its putative antigen, EBAG9/RCAS1, as a tumor marker. BMC Cancer 2005, 5:47.

33. Leelawat $K$, Watanabe T, Nakajima M, Tujinda S, Suthipintawong C, Leardkamolkarn V: Upregulation of tumour associated antigen RCAS1 is implicated in high stages of colorectal cancer. J Clin Pathol 2003, 56:764-768.

34. Giaginis C, Margeli A, Kouraklis G, Zira A, Tsourouflis G, Theocharis S: Diagnostic and prognostic utility of serum receptor-binding cancer antigen expressed on SiSo cells (RCAS1) levels in colon cancer patients. Int J Biol Markers 2009, 24:70-76.

35. Sonoda K, Miyamoto S, Kobayashi H, Ogawa S, Okugawa K, Taniquchi S, Wake N: The level of RCAS1 expression is inversely correlated with the number of vimentin-positive stromal cells in epithelial ovarian cancer. Int J Gynecol Cancer 2009, 19:838-843.

36. Sonoda K, Miyamoto S, Yamazaki A, Kobayashi H, Nakashima M, Mekada E, Wake N: Biologic significance of receptor-binding cancer antigen expressed on SiSo cells (RCAS1) as a pivotal regulator of tumor growth through angiogenesis in human uterine cancer. Cancer 2007, 110:1979-1990.

doi:10.1186/1479-5876-12-112

Cite this article as: Tanaka et al: Apoptotic function of tumor-associated antigen RCAS1 in oral squamous cell carcinoma. Journal of Translational Medicine 2014 12:112.

\section{Submit your next manuscript to BioMed Central and take full advantage of:}

- Convenient online submission

- Thorough peer review

- No space constraints or color figure charges

- Immediate publication on acceptance

- Inclusion in PubMed, CAS, Scopus and Google Scholar

- Research which is freely available for redistribution 